\title{
Index
}

Notes: 'n.' after a page reference indicates the number of a note on that page (e.g. 95n.3). References to tables are given in italics.

Abu Youssef, J. 139-40, 146 accommodation see housing Action for Refugee Women (AfRW) 146, 150n.8, 157-8, 168-9

activism, political: level of: migrant women 152-3

ADEMAF - Benin 147

Adshead, Kay 95n.7

AFAVO (Association des Femmes Africaines du Val d'Oise) 148

African (sub-Saharan) women's associations 147-8

Agence Nationale de l'Accueil des Etrangers et des Migrations (ANAEM) 97, 106, 117, 127n.1

Agence Nationale pour l'Emploi (ANPE) 117

Agence Nationale pour la Cohésion Sociale et l'Egalité de Chances (ACSE) 117, 128n.8

Ager, A. 31-2

Agrali S. 113

Ahmed, M. 73

aid, humanitarian 14

aide médicale d'état 43, 110

Albert, S. 96

Aliens Act (1905) 23

allocation d'insertion 43

allocation temporaire d'attente 43, 106

Amnesty International 2, 29, 86, 146
Section Française 41, 42, 97, 104, 146

Anafé (Association Nationale

d'Assistance aux Frontières pour les

Etrangers) 104, 106, 159

APIs (Asylum Policy Instructions) 35, 37 appeals for refugee status 25-7

Arab Women's Association (Birmingham) 140

Araujo, A. M. 138

arrival procedure

Britain 75-81

France 99-104

ASFAD 145, 146

Ashton, Catherine (Baroness) 37

Association d'Accueil aux Médecins et

Personnels de Santé Réfugiés 118

Association de Solidarité avec les

Femmes Algériennes et Démocrates 145, 146

Association de Solidarité avec les

Travailleurs Immigrés, Valence 1

Association Law (1901) 141, 142

Association Minkowska 112

Association pour les Victimes de la

Répression en Exil 112

Association Primo Lévy 97, 112

associations, refugee 2, 3, 5, 12n.1, 33,

129-51, 176-7

women's $22,137-51$

funding 141, 142-3

associations, voluntary, and social

cohesion 31, 143

ASTI,Valence 1

asylum: European Community

legislation 19-23 
Asylum Act (France, 1998) 39, 40

Asylum Aid 38, 79, 86, 150n.7, 157

Asylum and Immigration (Treatment of Claimants etc.) Act (2004) 24

Asylum and Immigration Act (1996) 24, 89

Asylum and Immigration Appeals Act (1993) 24

Asylum and Immigration Tribunal 26, 36 asylum appeals 2

Britain 38-9

France 41-2, 99

asylum applications: gender guidelines 34-8

asylum claims

statistics 61-4

Britain

destination of claimants 63

origins of claimants $62-5$

France

destination of claimants 64

origins of claimants $63-4,68,70$

asylum legislation: history

Britain 23-39

France 39-46

asylum policies

gender bias, studies 3

migration flows, impact on 51-2

Asylum Policy Instructions (2004) 35, 37

asylum procedures: access to 41

Britain 38-9, 75-81

France 39-42

asylum seeker: definition 8-9

asylum seekers: hostility to and fear of 32

Asylum Statistics (Home Office) 64-6

Atelier Grand Lyon - PLH 111

Athwal, H. 30

Attwood, C. 135-6, 150n.5

autorisation provisoire de séjour (APS) 99

babies: Britain: detention 83

Bacci, M. L. 50

Bade, K. 50

Bail for Immigration Detainees 74, 82

Baldwin, T. 31

Barab, S. A. 98

Barkham, P. 130

Barnett. M. 14

Bassel, L. 4, 98

BBC News 88, 91

Belluz, J. 84 benefits, welfare: legislation

Britain 27-9

France 42-4

Bernadot, M. 104

Bertheleu, H. 96

Bertrand, D. 98

Beski, C. 138

Bézier CADA 159

Bhabha, J. 3, 53

Bien-être de la Femme et de l'Enfant Congo Démocratique 140

Birnbery Peirce and Partners 88

Black Women's Network 134

Black, S. 51

Blais, A. 169n.1

Blake, J. 27

Bloch, A. 3, 4, 32, 74, 88, 89-90, 91, 94, 153

boat people 59

Bogus Woman, The (Adshead) 95n.7

Bohning, W. R. 50

bombings, London (July 2007) 31

Booth, H. 50

Border Agency, UK 124-5 culture of disbelief 173

Bosnia-Herzogovina 41

Bouaoumeur, Louisa 103

Boumedienne-Thierry, Alima 98

Bourne, J. 30

Bowcott, O. 31

Boyle, P. 53

British Medical Association 91

British National Party 78

British Nationality Act (1948) 54

'Britishness' 6, 30, 31, 47

Brocard, L. 45, 46

Buijs, G. 53

Building Cohesive Communities (Denham, 2001) 31

Butler, C. 92

Byrne, Liam 83

CADA 42-3, 106-9, 110-11, 113, 115, 118,127 n. $5-6$

CADAC (Coordination des Associations pour le Droit à l'avortement) 146

CAFDA (Paris) 111

Callamard, A. 3

Campani, G. 4

Canada: Gender Guidelines 35

Cantle Report (2001) 31 
Carriers' Liability Act (1987) 24

carriers' sanctions 41

Carter, R. 54

caseworkers: asylum procedure 173

France 101-4

Castles, S. 8, 31, 50, 51

Catani, M. 134

Catarino, C. $127 \mathrm{n} .2$

\section{CCIPH 85}

CEME (Commission des Eglises auprès des Migrants en Europe) 159

Ceneda, S. 36-7, 67, 82

Centre d'Etude de Documentation et de Recherches 99-100, 102

Centre de Formation Journalistes 118-19

Centre de Recherches Politiques, Sorbonne 98

Centres d'Accueil pour les Demandeurs d'Asile 42-3, 106-9, 110-11, 113, 115, 118, 127n.5-6

Centres de rétention administratives 104-6

Centres Médico-Psychologiques 111-12

Centres Provisoires d'Hébergement 108-9, 111, 113, 115

Cercle des Femmes Brésiliennes 137

CERE (Conseil Européen sur les Réfugiés et les Exilés) see ECRE

CESEDA 39, 40, 41

CFDT 117

CGT 117

Chaib, S. 117

Charlaff, L. 89, 91-2, 93

Chemiller-Gendreau, M. 96

Chenais, J. 51

childbearing and exile 113-14

children: education: France 119-21

Chile: migrants to France 59

Chuberre, M. 104

Churchill, Winston 54

Cimade, la 97, 105, 106, 128n.9, 159-60, 169

citizenship 129-51 concepts of 131-3

participation in civil society and $164-5$

Cleary, T. 82

CNDA 99

CNT 117
Code on the Admission and Residence of Foreigners and the Right to Asylum 39, 40, 41

cohesion, social 31, 143

Collard, S. 51

Collectif des Femmes Chilliennes 137

colonies: migration from (1945-75) 50-1

Comede 7, 112, 113-14

Comité d'Aide Exceptionelle aux Intellectuels Réfugiés 118

Comité de suivi des lois sur l'immigration $98,103,127$ n.2

Comité des femmes Djiboutiennes contre les viols et l'impunité 140

Comité interministériel de contrôle de l'immigration 42

Commission des Recours des Réfugiés 39, 45-6, 99

Commission for Citizenship 132

Commission for Patient and Public Involvement in Health 85

Commission for Racial Equality 31

Commission Nationale d'Admission (CNA) 107

Common Agenda for Integration, A (European Commission 2005) 22

Commonwealth Immigrants Act (1962) 24,54

Commonwealth, New: migrants to Britain 53, 54

Commonwealth, Old 71n.3

community advancement, refugee: refugee women $144-5,146-7$, 176-7

community associations, refugee $2,3,5$, 12n.1, 33, 129-51, 176-7

community cohesion 78

community relations: refugees: Britain 94

comparative analysis: British and French situation 124-7

Confédération Française Démocratique du Travail 117

Confédération Générale du Travail 117

Confédération Nationale du Travail 117

confidentiality: in asylum procedures 23

Conseil de l'Ordre des Médecins 110

Conseil Représentatif des Associations

Noires 123, 128n.10-11

Constitution, French (1793) 39

Constitution, French (1946) 39

Constitutional Act (1993) 40 
Contract of Reception and Integration $44-5,47$

contributory citizenship 131-2

Controlling our borders (2005) 26-7

Convention against Torture and other Cruel and Inhuman or Degrading Treatment or Punishment 15

Cooke, V. 134-40

Coordination de l'accueil des familles demandeuses d'asile (Paris) 111

Coordination Française pour le Droit d'Asile 159

Cornwall, A. 135, 147, 150n.4

Council for Assisting Refugee Academics 91

Council of Europe 29, 30

Directive 2003/9/EC 30

Recommendations 1478 and 1732 (2006) 22

countries of origin: information on 37

Cour nationale du droit d'asile 42

Couverture Médicale Universelle (CMU) 43, 110

CPH 108-9, 111, 113, 115

Crawley, H. 3, 15, 18, 22, 28, 29, 34, $35-6,38,53$

Credible Witness (Wertenbaker) 95n.7

credit unions 147-8

Crick, B. 132

Crick Report (2003) 31

Cris d'Exil 1

Crying Shame, $A$ (McLeish et al.) 83

Cutler, S. 3-4, 81-2

Dag, Firsat 78

D'Avanzo, C. 98

Davis, S. 134, 140

Deakin, N. 54

Delafontaine Hospital (St Denis) 114

Denham Report (2001) 31

Department of Health 85

Department of Work and Pensions 74

dependency: on spouses $64-5,130$

deportation

from Britain 88

from France 106

destination: choice of: asylum seekers:

France 100-1

destination society enrichment: refugee women's associations 143-4, 145-6 destitution: women asylum seekers:

Britain 80-1

detention 29-30

DFT (detained fast-track) 29-30, 81-2, 174

racism by staff: France 123

waiting zones 40,174

women asylum seekers: Britain 76, 81-3

women asylum seekers: France 104-6

Dhabo 140

Dietz, Mary 132, 133, 150n.3

Direct Airside Transit Visas 25

Discretionary Leave 27, 82

dispersal

Britain 28, 63

impact on political activity level

153

women asylum seekers 76-7, 174-5

health issues 86

labour market and 89

maternity 87

women's associations 139

France 43

displacement (1945-75) 50

dispositif national d'accueil 104

Diversity Equality Scheme and Action Plan (UKBA) 38

divorce: France 121-2

Dobson, J. 55, 56

Doctors for Human Rights 86

documentation: legislation on $25,27,38$, 89

domestic violence see violence, domestic

domestic work, illegal: France 115-16

Domingues, C. 100-1

Drake, H. 51

Dublin Convention 18, 19, 40, 43. $107-8,110$

Dublin Regulation see Dublin Convention

Dumes, A. 151 n.13

Dumon, W. A. 53

Dumper, H. 3, 26, 36, 64, 67, 73, 75, $76-7,78,81,84,86,88,90,92,93$, 94,95 n.3, 145,156

ECHR see European Convention on Human Rights

economic migrants 14

ECRE 20, 22, 74, 159, 169-70n.1 
education

access: France 118-21

level of: and work: refugee women:

France 116

skills and: refugee women: London 64, 67,145

Education sans Frontières 120

Electoral Commission, UK 152-3

ELELE 146

ELENA 20

Elle-Fondation 118-19

Elshtain, J. 132

Employability Forum 33, 74, 93

employment

participation in NGOs and 163

Britain 89-93

restrictions 27

services: stakeholder partnerships 33

France 115-17

empowerment: by NGOs 155, 163-6

entry controls: asylum seekers: Britain 24-5

Equal fund 117, 118, 119

Erel, U. 163, 168

étrangers: definition 9

Etudes sans Frontières 119

Eurodac 18

European Commission against Racism and Intolerance 122-3

European Commission of Social Rights 43

European Community legislation: asylum 19-23

European Convention on Human Rights 8, 26, 28, 40, 104, 124

breaches $28,34,83$

sans papiers and 60

European Council Meeting on Justice and Home Affairs 19

European Council on Refugees and Exiles 20, 22, 74, 169-70n.1

European Fund for Integration 48n.3

European Ministerial Conference on Integration (Vichy, 2008) 21

European Network against Racism 123

European Pact on Immigration and Asylum (2008) 21

European Refugee Fund 117

European Social Forums 177

European Union: free movement within 130
European Union Monitoring Centre on Racism and Xenophobia 123

European Women's Lobby 23

'Europeanness' 6, 130-1

Eveil, L' 137

Eversley, J. 92

Exceptional Leave to Remain 27, 169-70n.1

Express, L' 101-2

FAAR 118, 128n.9

families: refugees: Britain 94

Family Act (1996) 95n.10

family migration

disregarded by social scientists 53

to Britain 55-6

to France 58, 59-60, 61

family reunification: France 122

family reunification migration (post1975) 51-2

Farés, S. 107

Fassman, H. 50, 51

FASTI 143, 160-1

fast-track schemes: asylum claims 25,38 , 41-2

detained 29-30, 81-2, 174

Favell, A. 51

Fédération des Associations de Solidarité avec les Travailleurs Immigrés 143, $160-1$

Fédération Nationale des Associations pour l'Enseignement 7

Fédération Nationale Solidarité Femmes 121

Fédération Syndicale Unitaire 117, 128n.7

Feldman, N. 97-8, 134

feminist context 6

Femmes Bazouaises de France et de Cameroun 140

Femmes en Résistance 98

flight, internal: alternative to asylum 36

forced marriage 2, 45-6

Formation, Accueil des demandeurs d'Asile et Refugiés (FAAR) 118, 128n.9

Formation des Travailleurs Immigrés et de Leurs Familles 7

Fornah v. Secretary of State for the Home Department (2005) 36

Fortress Europe 5-6 
Forum Réfugiés 109, 111, 117

Français Musulmans d'Algérie (FMA) 58-9

France Terre d'Asile 7, 42, 44, 106

Freedman, J. 4, 97, 98

French Sociological Association 98

'Frenchness' 6

Front National 44

FSU 117, 128n.7

FTDA 107, 108, 109, 117, 127n.6

Full and Equal Citizens (2001) 31, 32

Fyvie, C. 56

Galvin, T. 3

Gantin, K. 98

Gateway Protection Programme 33

Gbadamassi, F. 116

Geddes, A. 20, 50

Gender Equality Duty 38

Gender Guidelines

Home Office 75, 125, 156

Immigration Appellate Authority $34-5,37$

UNHCR 16-17, 22, 23, 48, 126

Gender Issues in the Asylum Claim (2004) 35

gender neutrality, false: RSD processes:

France 125-6

gender related persecution see persecution, gender related

Geneva Convention (1951) 8, 10,13-15, 19, 20, 21-2, 24, 46, 48n.2, 99, 100, 104, 124

asylum decisions and: Britain 36-7

asylum decisions and: France 104

Cessation Clause 48n.4

ratification by Britain 24

ratification by France 40

genital mutilation, female 2, 36, 45-6, 48 n. 7

maternity care and 86,87

GFAMS 146

Gill, M. 28, 29

Gil-Robles, Álvaro 40, 41, 43

Giovannoni, L. 42

GISTI 118, 146

Goff, C. 143

Goulbourne, H. 139

Gould, C. 132-3

GRAF 46, 146, 169

Greater London Authority 64, 67, 91,
145

Greenslade, R. 78

Griffiths, D. 5, 28, 31, 32, 134

Groupe Accueil et Solidarité 7

Groupe Asile Femmes (le Graf) 46, 146, 169

Groupe d'Information et de Soutien des Immigrés 118, 146

Groupe Femmes Algériennes 137

Groupe Femmes pour l'Abolition des Mutilations Sexuelles 146

Guardian, The 74, 83, 89

Gueguen, M. 45-6, 160

Guidelines on International Protection: Gender Related Persecution

(UNHCR, 2002) 48n.2

Guidelines on the Protection of Refugee

Women (UNHCR, 1991) 48n.2

Gynécologie sans Frontières 115

Hague Programme 20-1

Halfacree, K. 53

Hammarberg Thomas 30

harm, private: distinguished from persecution 16

Harmondsworth immigration removal centre 29-30

HARP 85, 95n.12

Harrell-Bond, B. 3

Harris, C. 54

Harris, Hermione 86

Hathaway, James: hierarchy of rights 15

Hautefeuille CADA 111

headscarves 44

health care: access to 43

Britain 83-7 detention centres 82

France 110-15

Health for Asylum Seekers and Refugees Portal (HARP) project 85, 95n.12

health services: in detention and removal centres 30

High Council for Integration 9, 44

High Court: ruling on health care entitlement (2006) 84

Hinsliff, G. 34

Home Office 74

culture of disbelief 37

skills audit (2001) 90-1, 92

'honour' crimes 2, 45, 46

Hopwood, F. 78 
Horn of Africa Somali Women's

Organisation 146

Hortefeux, Loi 40

hostels: women asylum seekers: Britain 77

Housing Act (1996) 95n.10

housing

benefit 42

refugees: France 108-9

women asylum seekers

Britain 76-8, 79, 81

France 106-9

women refugees $174-5$

Hughes, Beverley 36

Human Rights Act (1998) 24, 26

Human Rights Watch: attestation of rape in Uganda 2

Humanitarian Protection 27, 33, 82, 169-70n.1

Hunt, L. 4

ICAR 36

identity documents 38

IGAS 110

IKWRO 146

ILPA 82

immigrant: definition 8-9

Immigration and Asylum Act (1999) 24, 28, 29, 32, 89

Immigration and Nationality Directorate 24

Immigration Appellate Authority Gender Guidelines 34-5, 37

immigration choisie 2

immigration policy: studies of gender bias 3-4

Immigration Rights Project 73

Immigration Status Documents 33

Immigration, Asylum and Nationality Act (2006) 24

immigration

interpreted as threat 18

to Britain: quantitative evaluation 55

to France: quantitative evaluation 58

immigrés: definition 9

Indefinite Leave to Remain 27, 33

Independent Race and Refugee News

Network 148

Indra, D. 3, 53

Information Centre about Asylum and Refugees in the UK 73-4, 79
INSEE (Institut National de la

Statistique et des Etudes

Economiques) 59, 136-7

Institute for Employment Studies 74, 89, 93

integration $74,147,175$

measurement of 47

policies $21,31-4,44-5$

General Secretary for Integration 44

Integration Matters 32-3

Inter Service Migrants 100

Interministerial Integration Committee 44

internally displaced persons 14

International Covenant on Economic,

Social and Cultural Rights 15

International Women's Day 158

interpreters: access to

Britain 84

France 100

interpreters: right to denied 41

interviews: asylum process

Britain 75

France 101, 102-3

Iranian and Kurdish Women's Rights

Organisation 140-1

Ireland: refugees' voting rights 169-70n.1

Is it Safe Here? (Dumper) 67, 158

isolation: women asylum seekers: Britain 77,80

in detention 83

Jad, I. 165

Jaggar, A. 164, 165, 166

Jenkins, Roy 30

Jenkins, S. 134

Jobcentre Plus 33-4

Jochum, V. 164

Joly, D. 14, 15, 134

Joshi, P. 18, 21, 23, 54

journalism, training in: France 119

Kabila, Joseph: government of

Democratic Republic of Congo 1

Kachepa,Vera 130

Kirby, T. 27

Kirk, R. 90-1, 92, 145

Kofman, E. 53, 61, 134

Koslowski, R. 51 
Kosovan conflict: and domestic violence 79

Kovess, V. 111

Kurdistan Refugee Women's

Organisation 141, 148

Kyambi, S. 28

labour market: refugees 33-4, 89-90

demand (1945-75) 50

Britain 89-93

France 115-17

labour market: restructuring (post-1975) 51

Labour Market System (LMS) 33-4

labour migration 51

to Britain 53-5, 56

to France $57,58,59,60-1$

labour movement: France 116-17

Lacaze, F. 45, 46

laicité 44

language needs: asylum seekers 74,88 , 161-2, 175

detention 82,83

health care 84

language skills:

asylum claims 41-2

NGO participation and $163,167-8$ refugees 91

language tuition, access to $45,88,118$, 161-2

Latin American Women's Aid Refuge 141

Latin American Women's Rights Service 137, 138

Laurain, S. 134

Layton-Henry, Z. 134

Le Gall, Catherine 101-2

Le Hérou, A. 110

Le Pors, A. 96

Le Vésinet Hospital (Yvelines) 115

Lebon, A. 53

legal representation: access to 82

legislation, asylum: European

Community 19-23

legislation, restrictive 4

Lemercier, E. 134

Leprince, C. 114

lèse majesty 39

Lesselier, C. 5, 100-1, 138, 152

Lester, T. 34, 35-6, 38

Lettre d'Information du GAS, La 7
Leveau, R. 96, 134

Lévi, F. 53

L'Hostis, H. 112

Ligue de l'Enseignement 120

Ligue des Droits de l'Homme 120

Lister, R. 132

literacy rates 88

Livret du citoyen 132

Lloyd, C. 134

loans: integration loan scheme 33

Locaux de rétention administratives

$$
\text { (LRAs) 104-6 }
$$

Lochak, D. 44

Loescher, G. 14

loi de finances de 200643

loi Relative à l'Entrée et au Séjour des Etrangers en France et au Droit d'Asile 59-60

loi Sarkozy 40

London

bombings (July 2007) 31

refugee women: education and skills $64,67,145$

Mayor of 74

London Framework for Regional Employment and Skills Action 90

London Skills Commission 90

loneliness: women asylum seekers:

Britain 77, 83

Lovenduski, J. 152-3, 164

Lutz, H. 130-1

Maastricht Treaty 71n.2

Maghreb countries: migrants to France 58-9, 60-1

Maison des Journalistes 118-19

Making Women Visible 81, 156

MANA (Bordeaux) 113

managed migration 2

Marocaines en France, Les 137

marriage, forced 2, 45-6

Marshall, T. H. 131

Martin, S. 53

Masson, S. 111

Maternity Alliance 3-4, 74, 86-7

maternity care

Britain 86-7

France 113-15

Maux d'Exil 7

McLeish, J. 3-4, 81, 83, 86, 87, 95n.1

Mead, L. 150n.2 
Médecins du Monde 86, 111, 112, 113, 114

media

discourse on asylum and refugees 4-5

hostility to asylum seekers 78,79 , $172-3$

medical care see health care

Medical Foundation for the Care of Victims of Torture 85

Medical Practitioners Union 85-6

Meeting the Health Needs of Refugees and Asylum Seekers in the UK (DoH) 85

Melis, C. 134

mental health

Britain 85-6

France 110, 111-13

Messina, A. M. 50

Midlands Refugee Women's Association 140

migrants, economic 14

migration flows 50-2

Migration Information Source 74

Migration PoIicy Institute (MPI) 59

migration: feminisation of 52-3

Miller, M. J. 50, 51, 153

Minister for Foreign Affairs 42

Ministry for Immigration, Integration, National Identity and Development 21,42

Missed Opportunities 90

Mlloja, Blerim 27

Moch, L. P. 50

Morokvasic, M. 53, 127n.2

Morris, T. 19

Mothers in Exile (Maternity Alliance) 86 mothers: asylum process and

Britain 77

France 102, 108

Mouffe, Chantal 132, 133

Mouterde, F. 132

MPSG 46

legal definition 35

MRAP 120, 124

MRWA 79

Münz, R. 50, 51

Muslim communities: Britain 30

Muslim women: vulnerability 78

Muslims: French Algerian 58-9

Musulmans, Français, d'Algérie (FMA) 58-9

muticulturalism: arguments against 31
Najjemba, Rose 36

Nantois, B. 134

National Asylum Support Service (NASS) 28, 74, 76-7, 78, 79, 80, 81, 158

National Insurance numbers 33

National Refugee Integration Forum 32, 33

National Union of Journalists 79

Nationality Act (2003) 130

Nationality Code 60

Nationality, Immigration and Asylum Act (2002) 24, 26-7, 28, 29

Nettleton, C. 15

New and the Old, The (Crick, 2003) 31

New Asylum Model (2005) 26-7

Quality Team 82

New Commonwealth: migrants to Britain 53, 54

New Right: conceptualisation of citizenship 131-2

New York Protocol relating to the Status of Refugees (1967) 13, 40

Newham Refugees Forum 95n.6

NGOs

comparative impact: Britain and France 47

refugee women's participation $161-8$, 176-7

NIACE 90

Noiriel, G. 96

non-refoulement: principle of 14, 20

Nygothi, Kamwaura 78

Oakington reception centre 26

Oasis (Observatoire pour l'Accueil des demandeurs d'asile) 118, 128n.9

Offences Against the Person Act (1861) 95n. 10

Office Central pour la Répression du Trafic des Etres Humains (OCRTEH) 116

Office for Criminal Justice Reform 76

Office Français de Protection des Réfugiés et Apatrides see OFPRA

Office National d'Immigration 57

OFPRA 38, 39-42, 45, 46, 63, 67-71, 98, 99-104, 106, 107, 108, 124-5, 126

culture of disbelief 100,173

oil crisis (1973-74) 51 
Old Commonwealth 71n.3

OMCT 105

'one-stop appeal': asylum claims 25

opinions, political: basis for refugee status 46

Owers, Anne 82

Oxfam 19-20, 74, 79, 80, 155

Palestinian women in Britain 139-40, 146

Palidda, S. 134

Palmer, C. $36-7$

Parekh, B. 164, 165

Paris-Dépôt CRA 105-6

Parmar, P. 134

Parti Socialiste 44

participation, civic: statistics 135-6

parties, political: involvement of migrants 153

Pasqua law (1993) 60

passports 38

Pateman, C. 132

Pearl, M. 134

persecution

definition 15, 16, 17

fear of 13-14

sexual orientation or gender grounds 19

gender related 16-18, 19, 22-3, 35-6, $37,45-6,129-30,159-60$

medical evidence 102

see also rape; sexual violence; torture non-state agents 19, 22, 34, 35, 36, 37

Phillips, Anne 132, 133

Phillips, Trevor 31

Phizacklea, A. 53

Piore, M. J. 50

Plant, R. 150n.2

police: racism: France 123

policy: refugee and asylum: ideological aspects 13

political activism: level of

migrants 153

women 152-3

political opinions: basis for refugee status 46

political participation

concepts of 133

cultural issues $167-8$

political refugees (1945-75) 51

Poppy project 76 population, refugee

proportion in Europe 2

proportion of women 2

poverty: women asylum seekers: Britain

80-1

Praxis Community Projects 92

préfectures: fast-tracking asylum claims

41

pregnant women: asylum process and:

Britain 76-7

detention 83

press: hostility to asylum seekers 78,79 , $172-3$

Proasile 7

Procedures Directive, European

Community (2005) 19-20

gender issues 22-3

prostitution: France 116

Protection from Harassment Act (1997)

95n.10

PS (Parti Socialiste) 44

Qualification Directive, European

Community (2004) 19, 20, 37

gender issues 22

qualifications: refugees: Britain 90-3, 145

Quiminal, C. 134

racism

Britain

impact on women asylum seekers

78-9, 82

maternity services 86,87

NGOs 168

France $122-4,126-7$

schools 120

violence $126-7$

Rai, S. 166

Rajfire 45, 46, 95-6, 100, 116, 141, 145, 146

Randall, A. 66

Randall,V. 152

rape

ECHR and 36

Ugandan military 1-2

see also persecution, gender related

Rapoport, M. 96

RCO Women's News (Refugee Council) 156

Recchi, E. 51 
reception centres 30

asylum seekers: France 106-9

Reception Conditions Directive, European Community (2003) 19, 43

reception procedure $174-5$

Britain 75-81

Red Cross 76, 79, 86, 155

Reeves, K. 56

refoulement 106

Refugee Action 29, 74, 77, 79, 95n.3, 150n. 8, 157-8, 166

Refugee Convention (1951) see Geneva Convention (1951)

Refugee Council 19, 27, 34, 38, 74, 76, 79, 80-1, 86, 87, 91, 143, 155-6, $158,166,168$

Refugee Integration and Employment Service 33

Refugee Integration Strategy 32-3

Refugee Media Agency 79

refugee migration

to Britain 55,56

to France 58, 59, 71

Refugee or Person in need of International Protection (Qualification) Regulations (2006) 37

refugee status: determination procedures $25-7,124-7,173$

studies 4

France 99-104

refugee studies: academic field 4

Refugee Women and Domestic Violence (RWRP) 95n.9

refugee women: education and skills 64 , 67

Refugee Women's Association 74, 146, 150n.10

Refugee Women's Legal Group (RWLG) $34,35,154,156,157$

Refugee Women's News 7

Refugee Women's Resource Project 7, 21, 23, 28-9, 35, 36-7, 38-9, 74, 75, 79, 80, 82, 95n.9, 125, 146, 150n.7, 157

refugee: definition 8-9, 13-14, 19 gender issues 22

Refugees and Asylum Seekers Mass Media 79

refuges, women's 80

Regional Protection Programmes 20-1 relocation: alternative to asylum 36

Rémy, J. 101-2

repatriation programmes, 'voluntary' 14

research: on asylum seeking and refugee women: France 97-8

Réseau pour l'autonomie des femmes immigrées et réfugiées see Rajfire

RESEDA 59-60

resident's permit: requirements for 45

Revenu minimum d'insertion 44

Rex, J. 134

rights: James Hathaway's hierarchy of 15

rights, concepts of 132-3

Rigoni, I. 98

Robin, A. 150n.6

Robinson, D. 56

Robinson, V. 51

Roche, M. 150n.2

Roma women: pregnancy: in France 114

Royal College of Nursing 93

Rozenberg, G. 31

Rubin, J. 116

Rudge, P. 51

RWRP see Refugee Women's Resource Project

safe conduct pass 99

'safe countries' 19-20, 25, 26, 41

asylum seekers returned to 106

failure to protect women 38-9

trafficking from 75

Safe for Whom? (RWRP) 157

Salinas, M. 134

Salisbury, Marquis of 54

sans papiers $60-1,111,130,145$

Sarkozy, Nicolas 2-3

Savoirs et Formation 7

Schengen Agreement (1985) 18, 40

Schlenzka, N. 4

Schuster, L. 23, 24

Schwertz-Favrat, O. 1

Scottish Executive 91-2

Scottish Refugee Council 91-2

SDFE 122

Secours Catholique 97, 140

Secure Borders, Safe Haven (2001) 31

Ségur, P. 96

Séhili, D. 98

'selective' migration 2-3

sex work: France 116 
sexual harassment

detention 82

hostels, hotels and shared accommodation $77,87,95 n .1$

sexual orientation: persecution for 46

sexual violence see violence, sexual

Shah and Islam case (House of Lords, 1999) 35-6

Sharia law 31

shelter, right to 28

Shutter, S. 3, 53

Sigona, N. 28, 31, 32, 134

Siim, B. 132, 133

Simmonot, C. 104

Single European Act (1986) 18, 71n.2

single women: asylum process and

Britain 77-8

France 108

Sissoko, A. 134

skills: refugees: Britain 90-3, 145, 150n.6

social capital: NGO participation 163-6

Solomos, J. 23, 24

Somalis in Britain 86, 88, 92, 94

women $66-7,140$

Somerville, W. 48n.1, 73

Sommo, L. 4

SOS-Racisme 124

South Asian immigrants: rotation system 55

Spijkerboer, T. 3

Sriskandarajah, D. 78

Stancer, C. 3-4

state: relationship to citizens 131-2, 148-9

State Medical Aid (AME) 43

statistics

asylum seekers and refugees: Britain: qualifications 92

civic participation 135-6

dependent spouses 130

gender disaggregated 125, 126

lack of 49-50

immigration to Britain 54, 55, 56

immigration to France 57-9, 61

migration: by gender 52

Britain 64-7

France $67-70$

racism: France 123

refugees: Britain: employment levels:

89 refugees: London: women: education and training 145

women's use of NGO services 156

Stewart, J. 42

Strang, A. 31-2

Strasbourg CADA 111

subsidiary protection $45,169-70$ n.1

Sudan Women's Association 146

Sudbury, J. 134

suicide: in detention and removal centres 30

Sukula, Flores 81

Sunrise (Strategic Upgrade of National Refugee Integration Services) 32-3

Taboa-Leonetti, I. 53

Tait, K. 90, 91, 93

Temporary Protection 48n.5

terminology 8-9

territorial asylum 40

Thatcher, Margaret 24

They Took Me Away (RWRP) 82, 157

Thiara, R. K. 134

Tiberghien, F. 96

Tomlinson, F. 163, 168

Tomlinson, S. 134

tontines $147-8,151 \mathrm{n} .13$

torture

reason for asylum claims 82

risk of 15

Ugandan military 1

see also persecution, gender related

trade unions: France 116-17

trafficked women: asylum procedure and

Britain 75, 76

France 116

trafficking, human 45

transit centres: France 109

transit visas 41

Traumatic Stress Clinic 85

'Traumatised Refugees in Europe' (EU Commission funded project) 8

Tribalat, M. 58-9

UCIJ 41

Uganda: 'safe-houses' 1

UK Border Agency 124-5

culture of disbelief 173

UK Border and Immigration Agency 24

UK Electoral Commission 152-3 
UMP (Union pour un Mouvement

Populaire) 2-3

unemployment: refugees 33-4

Union pour un Mouvement Populaire 2-3

Union Syndicales Solidaires 117, 128n.7

United Nations Committee against Torture 48n.8

United Nations Conference on Women (Beijing, 1995) 73

United Nations High Commission for Refugees (UNHCR) 2, 14, 15, 75, 83,104

Gender Guidelines 16-17, 22, 23, 48, 126

Handbook 15

Universal Declaration of Human Rights 15

Universal Medical Coverage 43

universalism, republican 47, 172

NGOs and 169

University of East London 95n.6

USS 117,128 n.7

Valluy J. 4, 98

Vasquez, A. 138

Veith, B. 134

Vertovec, S. 134

Viché, C. 134

Vie Privée et Familiale 59-60

Villepin, Loi de 40

violence, domestic 95n.8-10

Britain 75, 79-80

France 45, 121, 159

violence, gender-related: as persecution 16-18, 22-3, 36, 37

violence, racist $126-7$

violence, sexual

reason for asylum claims 82

see also persecution, gender related

violence: against asylum seekers: Britain 78

deportations 88

violence: detention centres: France 105

visa requirements 25

Vogel, U. 132

voluntary associations: and social cohesion 31,143

voluntary sector:

integration and 32, 33-4, 163-5

refugee women's participation $161-8$ vote: right to $169-70 \mathrm{n} .1$ voucher system 28

Wadia, K. 4, 67, 88, 98, 99, 134

wages: refugees: Britain 89-90

Wahlbeck, O. 134

waiting zones 40, 99, 174

Wales Media Forum 79

Wallace, T. 50

WAN see Women's Asylum News

WAR (Women Against Rape) 36

Wareham, Rachel 79

Watson, T. 56

Weaver, M. 83

welfare benefits: legislation

Britain 27-9

France 42-4

Werbner, P. 132, 139

Wertenbaker, Timberlake 95n.7

Wetherill, B. 132

widowhood rites 46

Wihtol de Wenden, C. 96, 134

Wilpert, C. 134

Wluczka, M. 111

Women Against Rape 36

Women Asylum Seekers in the UK (RWRP) 157

Women Asylum Seekers Together 141

Women, Doing it for Ourselves (Refugee Council) 156

Women for Refugee Women 141, 146, 150n.9

Women's Asylum News 7, 29, 37, 82, 83, $95 \mathrm{n} .1,157$

women's refuges 80

work, right to $42,89,115$

Working Lives Research Institute 73-4, 168

Working to Rebuild Lives (DWP) 33

World Health Organisation 74, 84-5

Wyman, M. 50

Yarl's Wood immigration removal centre $29-30,75,76,81,82,88$

Young, I. 132, 133

Yuval-Davis, N. 132

ZAPI-3 106

Zetter, R. 28, 31, 32, 43, 134

Zolberg, A. R. 50

zones d'éducation prioritaire 119-20

zones d'attente 40, 99, 174 